PSICOLOGIA, SAÚDE \& DOENÇAS, 2018, 19(2), 337-353

ISSN - 2182-8407

Sociedade Portuguesa de Psicologia da Saúde - SPPS - www.sp-ps.pt

DOI: http://dx.doi.org/10.15309/18psd190214

\title{
PREVENÇÃO DO TABAGISMO NA ESCOLA: AVALIAÇÃO DE UM PROGRAMA BASEADO NO CURRÍCULO
}

Isabel Sousa (isabelsousa@ie.uminho.pt)

Instituto de Educação, Universidade do Minho, Campus de Gualtar, 4700-057 Braga, Portugal.

\section{PREVENÇÃO DO TABAGISMO NA ESCOLA: AVALIAÇÃO DE UM PROGRAMA BASEADO NO CURRÍCULO}

RESUMO: O consumo de tabaco é um problema de saúde pública com graves consequências para a saúde dos indivíduos. $\mathrm{O}$ início do consumo ocorre predominantemente na adolescência, o que justifica intervenções em meio escolar. Este estudo propõe-se avaliar a eficácia de um programa baseado no currículo escolar, "SmokeOut-II", na melhoria dos conhecimentos e crenças sobre tabagismo e no comportamento face ao consumo de tabaco em jovens escolarizados no $9^{\circ}$ ano do Ensino Básico, por sexo. Trata-se de um estudo quasi-experimental, do tipo pré/pós-teste, com um grupo experimental $(n=159)$ e um grupo de controlo $(n=171)$, realizado no ano letivo de 2014/2015. Aplicou-se um questionário, em ambos os grupos, seguido da intervenção (programa "SmokeOut-II") no grupo experimental. Aplicou-se, no pós-teste, o mesmo questionário a ambos os grupos e compararam-se os resultados, usando estatística inferencial. Verificou-se no grupo experimental uma melhoria, entre o pré e o pós-teste, nos conhecimentos e uma mudança positiva em algumas crenças sobre tabagismo, além de um decréscimo no consumo de tabaco nos rapazes. A iniciação e a experimentação do consumo de tabaco diminuíram no grupo experimental, destacando-se nos rapazes a intenção de experimentar fumar no mês seguinte e nas raparigas a intenção de fumar no ano seguinte, mantendo-se esta tendência antes e depois dos 18 anos. O programa melhorou os conhecimentos e as crenças sobre tabagismo, tendo sido benéfico, a curto prazo, na prevenção da experimentação e na intenção de vir a fumar, bem como na iniciação e consumo. Será fundamental estudar os efeitos do programa a longo prazo.

Palavras-chave: tabagismo, saúde infantil, prevenção tabágica, psicologia preventiva

\section{SMOKING PREVENTION IN SCHOOLS: EVALUATION OF A PROGRAMME BASED ON THE CURRICULUM}

ABSTRACT: Tobacco use is a public health problem with serious consequences for the health of individuals. The onset of consumption mainly occurs in adolescence, which calls for school-based interventions. This study intends to evaluate the effectiveness of a programme based on the school curriculum,

Campus de Gualtar; 4710-057 Braga, Portugal. e-mail:isabelsousa@ie.uminho.pt 


\section{PREVENÇÃO DO TABAGISMO NA ESCOLA}

"SmokeOut-II", in the improvement of knowledge and views about smoking as well as in the smoking behavior among adolescents in the $9^{\text {th }}$ year of Basic Education, by sex. This is a quasi-experimental, pre and post-test type study, with an experimental $(n=159)$ and a control group $(n=171)$, held in $2014 / 2015$. A questionnaire was applied to both groups, followed by the intervention ("SmokeOut-II" program) in the experimental group. In the post-test, the same questionnaire was applied to both groups and the results were compared using inferential statistics. In the experimental group, there was an improvement in knowledge and a positive change in some views about smoking, in addition to a decrease in the use of tobacco among boys. The initiation and experimentation of smoking decreased in the experimental group, mostly the boys' intention to try smoking the following month and the girls' intention to smoke the following year. This tendency continued before and after the age of 18. The program has improved knowledge and positively changed some views about smoking; it has been beneficial in the shortterm prevention of experimentation and intention to smoke, as well as in initiation and tobacco consumption. It is essential to study the long-term effects of the programme.

Keywords: smoking, children's health, smoking prevention, preventive psychology

Recebido em 14 de Março de 2018 / Aceite em 24 de Maio de 2018

Uma em cada 10 mortes em todo o mundo é causada pelo uso de tabaco (WHO, 2017). O consumo de tabaco é uma epidemia global entre os jovens, sendo consumido por crianças e adolescentes, o que acarreta um risco elevado de dependência desta substância (USDHHS, 2012). Prevê-se que 5,6 milhões de crianças com menos de 18 anos morram prematuramente em consequência do tabagismo (USDHHS, 2014). Pelas suas consequências e elevada prevalência em crianças e adolescentes, o tabagismo é considerado um flagelo social, sendo necessário combate-lo precocemente, prevenindo a experimentação e o início do consumo de tabaco nas crianças e jovens.

Estudos efetuados em adolescentes revelam que o consumo de tabaco é iniciado muitas vezes na escola, em idade precoce, o que justifica intervenções didáticas e multidisciplinares ao longo do percurso escolar (Mercken et al., 2012). A escola é o meio ideal para intervir junto de crianças e jovens e promover educação em saúde, sendo a prevenção dos consumos, nomeadamente do tabaco, uma área fundamental (Precioso, Samorinha, \& Macedo, 2016). O desafio consiste em intensificar e adaptar, por sexo, intervenções que incidam sobre a prevenção da experimentação entre os adolescentes.

A evidência científica mostra que intervenções multicomponentes são eficazes na redução da prevalência do consumo de tabaco nos adolescentes, sendo os programas em meio escolar parte fundamental dessas intervenções (Mercken et al., 2012; Thomas, McLellan, \& Perera, 2013). Entre estes, os programas continuados que integram o contexto social têm-se mostrado eficazes (Andersen, Krølner, \& Bast, 2015; Nădăşan, Chirvăsuţă, \& Ábrám, 2015; Kanicka, Poniatowski, \& Szpak, 2013a). Em Portugal existem os programas "Não fumar é o que está a dar" (Precioso, 1999), dirigido a alunos do $7^{\circ}$ ano de escolaridade, "Querer é Poder I" (Vitória, Raposo, \& Peixoto, 2000) e "Querer é Poder II" (Vitória, Raposo, \& Peixoto, 2001), destinados a jovens dos 12 aos 14 anos. Apesar de terem demonstrado eficácia (Precioso, 2001; Vitória, Silva, \& De Vries, 2011), estes programas foram desenvolvidos sem um conhecimento aprofundado dos determinantes do consumo em função do sexo. Foi por isso importante melhorar e modernizar os programas existentes em Portugal, tendo em conta as diferenças de género, e avaliar o seu impacte a curto prazo. 
O programa "SmokeOut-II" é um programa multidisciplinar de prevenção do tabagismo, que tem como objetivos melhorar os conhecimentos e modificar crenças sobre o tabagismo, bem como diminuir o consumo de tabaco e a iniciação, a experimentação e a intenção de vir a fumar, reduzindo a sobrestima da prevalência do consumo de tabaco (Precioso et al., 2014). Foi concebido com base em programas já existentes e adaptado a alunos do $9^{\circ}$ ano do ensino básico, em função do género, sendo constituído por uma componente informativa e uma componente de competências sociais, incluindo estratégias de redução dos fatores de risco individuais e microssociais, promovendo fatores protetores, como a família e a escola, pela relevância que estes elementos têm no processo de socialização e integração dos jovens.

A literatura sobre programas educativos revela que estes se baseiam em teorias e modelos orientados para a compreensão dos comportamentos, sendo estes o resultado da intenção comportamental. Segundo a Teoria da Ação Racional, a intenção é mensurada pelas atitudes relativas a uma ação específica e pelas normas subjetivas (Fishbein, \& Ajzen, 1975). A atitude relativamente a um comportamento é influenciada pelas crenças sobre as consequências do ato e pela perceção que a pessoa tem dessas consequências. Assim, os adolescentes podem apresentar posturas distintas relativamente ao comportamento de fumar de acordo com a perceção que têm sobre as consequências desse ato em termos individuais, familiares e sociais. O modelo da Ação Racional tem fortes implicações no desenho de programas de prevenção do consumo de tabaco, sendo suposto que a promoção de atitudes favoráveis a não fumar e o desenvolvimento de uma norma subjetiva desfavorável ao consumo de tabaco possam ter um elevado efeito preventivo (Precioso, 2001).

Este programa propõe-se potenciar que os alunos adquiram conhecimentos sobre as consequências do consumo de tabaco no organismo e na saúde, obtenham competências para contrariar a sobrestima sobre o hábito de fumar, experimentem situações de treino para desenvolver capacidades de recusa de tabaco e desenvolvam estratégias para ajudar os colegas a deixar de fumar. Considerando a influência de fumar nos projetos de vida, pretende-se também que os alunos tomem uma decisão assertiva face ao comportamento de fumar para além de tomarem consciência dos custos associados à compra de tabaco. O programa aborda também os problemas associados à exposição ao Fumo Ambiental do Tabaco e propõe aos alunos a assinatura de um contrato de "Promessa de uma vida livre de fumo", comprometendo-os a não fumar no futuro. O programa "SmokeOut-II" foi desenhado para ser aplicado em contexto escolar, na sala de aula.

Este estudo tem como objetivo avaliar a eficácia do programa "SmokeOut-II" na melhoria dos conhecimentos e crenças sobre tabagismo, na experimentação e intenção de vir a fumar, na iniciação e no consumo de tabaco em jovens escolarizados, a frequentar o $9^{\circ}$ ano do Ensino Básico, por sexo.

\section{MÉTODO}

\section{Participantes}

A amostra deste estudo é constituída por 330 alunos do $9^{\circ}$ ano do Ensino Básico, repartidos por um grupo experimental $(n=159)$ e um grupo de controlo $(n=171)$, realizado no ano letivo de 2014/2015. O grupo experimental era constituído por alunos de oito turmas pertencentes a dois agrupamentos de escolas do distrito de Braga (Agrupamento Sá de Miranda e Agrupamento de Vila Verde), sendo composto por 72 alunos do sexo masculino e 87 do sexo feminino, com idade média de 14,6 anos. $\mathrm{O}$ grupo de controlo era constituído por oito turmas de dois agrupamentos de escolas do distrito de Braga (Agrupamento D. Maria II e Agrupamento de Póvoa de Lanhoso), sendo composto por 91 alunos do sexo masculino e 80 do sexo feminino, com idade média de 14,7 anos. O nível socioeconómico dos 


\section{PREVENÇÃO DO TABAGISMO NA ESCOLA}

alunos em ambos os grupos era semelhante. A seleção das escolas foi feita por conveniência, através de um convite efetuado pelos investigadores aos Diretores dos respetivos agrupamentos. Os alunos de ambos os grupos tiveram autorização prévia dos Encarregados de Educação para a participação no estudo. Foi atribuído um código a cada participante, permitindo efetuar o seu emparelhamento entre o pré e o pós-teste.

\section{Material}

Aplicou-se um questionário de autopreenchimento anónimo construído com base em instrumentos já validados e utilizados em outros estudos sobre comportamento tabágico (Precioso, 2001; Currie, Hurrelmann, \& Settertobulte, 2000, 2004; Currie, Gabhainn, \& Godeau, 2008; Currie et al., 2012; Inchley et al., 2016; Precioso, \& Catarina, 2014).

O questionário era constituído por 23 questões de resposta múltipla que avaliavam: variáveis sociodemográficas (idade, sexo, local de residência, tipo de agregado familiar, escolaridade dos pais e nível socioeconómico), experimentação, intenção de experimentação, consumo, iniciação, dependência, cessação, intenção de cessação, exposição ao fumo ambiental do tabaco (FAT) e exposição no domicílio, norma subjetiva sobre o consumo de tabaco, abordagem sobre os malefícios do tabaco, capacidade de recusa, conhecimentos, ocupação de tempos livres, sentimento em relação à escola, intenção de saber mais sobre o tabaco, crenças sobre o tabagismo e a frequência de assuntos relacionados com tabaco.

\section{Procedimento}

Após autorização prévia da Direção-Geral de Inovação e Desenvolvimento Curricular do Ministério da Educação e dos respetivos Diretores dos Agrupamentos para a realização do estudo, foram contactados os professores coordenadores de Educação para a Saúde dos estabelecimentos de ensino e fornecidas indicações para homogeneizar a implementação do Programa. Foram selecionadas aleatoriamente em cada escola quatro turmas de $9^{\circ}$ ano. Nas escolas do grupo experimental foi implementado o programa "SmokeOut-II" por professores que receberam formação especializada para o efeito, na qual foram explicitados os objetivos do programa, estrutura, metodologia, resultados esperados e materiais necessários para a intervenção, de forma a aplicarem a intervenção com sucesso. Essa formação foi ministrada por formadores certificados e acreditada pelo Conselho Científico Pedagógico da Formação Contínua da Universidade do Minho, num curso de 25 horas. Após obtenção da autorização dos Encarregados de Educação, os questionários foram aplicados pelos Diretores de Turma ou pela bolseira do projeto de investigação.

Este estudo englobou um grupo experimental, no qual a investigação decorreu em três momentos distintos (pré-teste, intervenção e pós-teste) e um grupo de controlo que participou em dois momentos (pré-teste e pós-teste). O primeiro momento (pré-teste), comum aos dois grupos e realizado no primeiro período do ano letivo, consistiu na aplicação do questionário construído para o efeito. $\mathrm{O}$ segundo momento, que apenas ocorreu no grupo experimental, consistiu na implementação do programa "SmokeOut-II", pelos professores das disciplinas envolvidas, em contexto de sala de aula, durante o primeiro, segundo e terceiro período. No final do terceiro período, foi aplicado o mesmo questionário (pós-teste) utilizado no primeiro momento, em ambos os grupos, com o intuito de comparar os dois grupos entre o pré e o pós-teste e avaliar a eficácia do programa na prevenção do tabagismo. 


\section{Intervenção}

O programa "SmokeOut-II" é um programa de prevenção do tabagismo destinado a melhorar os conhecimentos e a modificar crenças sobre tabagismo, diminuir o consumo de tabaco e retardar a iniciação, bem como evitar a experimentação e minorar a intenção de vir a experimentar fumar tabaco, reduzindo a sobrestima sobre o comportamento de fumar. É direcionado a alunos do $3^{\circ}$ ciclo do Ensino Básico e foi desenhado para ser aplicado em sala de aula, pelos professores das disciplinas envolvidas. É constituído por catorze sessões (figura 1), segmentadas em: 1) Efeitos do consumo de tabaco na saúde; 2) Efeitos do consumo de tabaco na aparência e no organismo; 3) Composição do fumo do tabaco; 4) Problemas associados com a exposição ao fumo do tabaco; 5) Vantagens de não fumar para a mulher; 6) Fumar ainda estará na moda?; 7) Cálculo do gasto com a compra de tabaco; 8) O tabaco e o exercício físico; 9) Estilos de comunicação; 10) Fatores associados ao consumo de tabaco; 11) Ajudar os colegas a deixar de fumar; 12) Influência de fumar na concretização dos projetos de vida; 13) Formar uma opinião e tomar uma decisão sobre o comportamento de fumar; 14) Assinatura da declaração "Promessa de uma vida livre de fumo".

\begin{tabular}{|c|c|c|c|c|c|c|c|c|}
\hline \multirow[b]{2}{*}{$\begin{array}{l}N^{\mathbf{0}} \\
\text { Sessão }\end{array}$} & \multirow[b]{2}{*}{ Título da sessão } & \multicolumn{7}{|c|}{ Disciplinas } \\
\hline & & C.N. & F.Q. & Mat. & Port. & E. F. & E. V. & $\begin{array}{r}\text { Out } \\
\text { ras }\end{array}$ \\
\hline & Aplicação do Questionário Pré-teste & \multicolumn{7}{|c|}{ Diretor de Turma } \\
\hline 1 & Efeitos do consumo de tabaco na saúde. & $\mathrm{X}$ & & & & & & \\
\hline 2 & $\begin{array}{l}\text { Efeitos do consumo de tabaco na } \\
\text { aparência e no organismo. }\end{array}$ & & & & & & $\mathrm{X}$ & \\
\hline 3 & Composição do cigarro. & & $\mathrm{X}$ & & & & & \\
\hline 4 & $\begin{array}{l}\text { Problemas associados ao Fumo } \\
\text { Ambiental do Tabaco (FAT). }\end{array}$ & & $\mathrm{X}$ & & & & & \\
\hline 5 & Vantagens de não fumar para a mulher. & $\mathrm{X}$ & & & & & & \\
\hline 6 & Fumar ainda estará na moda? & & & $\mathrm{X}$ & & & & \\
\hline 7 & Cálculo do gasto com tabaco. & & & $\mathrm{X}$ & & & & \\
\hline 8 & O tabaco e o exercício físico. & & & & & $\mathrm{X}$ & & \\
\hline 9 & Estilos de comunicação. & & & & $\mathrm{X}$ & & & \\
\hline 10 & $\begin{array}{l}\text { Fatores associados ao consumo de } \\
\text { tabaco. }\end{array}$ & & & & & & & $\mathrm{X}$ \\
\hline 11 & Ajudar os colegas a deixar de fumar. & & & & & & & $\mathrm{X}$ \\
\hline 12 & Influência de fumar nos projetos de vida. & & & & & & & $\mathrm{X}$ \\
\hline 13 & $\begin{array}{l}\text { Formar uma opinião e tomar uma decisão } \\
\text { sobre o comportamento de fumar. }\end{array}$ & & & & & & & $\mathrm{X}$ \\
\hline \multirow[t]{2}{*}{14} & $\begin{array}{l}\text { Declaração Promessa de uma vida livre } \\
\text { do fumo do tabaco. }\end{array}$ & & & & & & & $\mathrm{X}$ \\
\hline & Aplicação do Questionário Pós-teste & \multicolumn{7}{|c|}{ Diretor de Turma } \\
\hline
\end{tabular}

Legenda: CN-Ciências Naturais; FQ-Físico-química; Mat-Matemática; Por-Português; EF- Educação Física; EV- Educação Visual.

Figura 1.

Sessões do Programa "SmokeOut-II" 


\section{PREVENÇÃO DO TABAGISMO NA ESCOLA}

\section{Análise estatística}

Para testar as associações entre os grupos experimental e de controlo, em cada momento, foi utilizado o Teste de Qui-Quadrado da Independência, na comparação do pré-teste com o pós-teste, recorreu-se ao Teste de McNemar. A análise estatística foi realizada com recurso ao IBM SPSS Statistics 24 .

\section{RESULTADOS}

\section{Conhecimentos sobre tabagismo}

$\mathrm{Na}$ análise dos conhecimentos sobre tabagismo (quadro 1), definiu-se como conhecimento adequado os alunos considerarem "prejudicial" ou "muito prejudicial" um determinado efeito do tabaco no organismo, por oposição às opções de resposta "nada prejudicial" e "pouco prejudicial". As percentagens apresentadas referem-se aos participantes que apresentaram conhecimentos adequados.

Globalmente, os alunos do grupo experimental apresentaram melhorias entre o pré e o pós-teste em ambos os sexos, comparativamente ao grupo de controlo. Constatou-se que a totalidade dos rapazes e raparigas do grupo experimental, no pós-teste, tinham a perceção de que o consumo de tabaco é prejudicial ou muito prejudicial para os pulmões e saúde em geral. Constatou-se ainda que, no grupo experimental, entre o pré e o pós-teste, houve um aumento estatisticamente significativo do conhecimento sobre os efeitos do tabagismo na sexualidade, tanto nos rapazes (de 70,4\% para 93,1\%; $\mathrm{p}<0,001$ ) como nas raparigas (de 53,6\% para 97,7\%; $\mathrm{p}<0,001$ ), e na pele, tanto nos rapazes (de $66,2 \%$ para 94,4\%; p<0,001) como nas raparigas (de 85,1\% para 98,9\%; $\mathrm{p}=0,001$ ). Analisando por sexo, verificou-se um aumento estatisticamente significativo, no grupo experimental, entre o pré e o pósteste, da percentagem de rapazes com a percepção de que o consumo de tabaco é prejudicial ou muito prejudicial para o coração (de $93,0 \%$ para $100 \%$; $=0,028$ ) e que afeta a capacidade de fazer desporto (de $93,0 \%$ para $100 \% ; \mathrm{p}=0,028$ ). 
Isabel Sousa

Quadro 1.

Conhecimentos sobre os efeitos do tabagismo no organismo, no pré e no pós-teste, por sexo

\begin{tabular}{|c|c|c|c|c|c|c|c|c|}
\hline \multirow{2}{*}{$\begin{array}{l}\text { O tabaco é } \\
\text { prejudicial para }\end{array}$} & \multirow[b]{2}{*}{ Sexo } & \multirow[b]{2}{*}{ Grupo } & \multicolumn{2}{|c|}{ Pré-teste } & \multicolumn{2}{|c|}{ Pós-teste } & \multicolumn{2}{|c|}{ Pré/Pós teste } \\
\hline & & & $\mathrm{N}$ & $\mathrm{n}(\%)$ & $\mathrm{N}$ & $\mathrm{n}(\%)$ & $x^{2}$ & $\mathrm{p}$ \\
\hline \multirow{4}{*}{ Pulmões } & \multirow{2}{*}{ Rapaz } & Controlo & 91 & $89(97,8)$ & 91 & $87(95,6)$ & 0,17 & 0,682 \\
\hline & & Experimental & 71 & $70(98,6)$ & 72 & $72(100)$ & 0,00 & 0,497 \\
\hline & \multirow{2}{*}{ Rapariga } & Controlo & 80 & $80(100)$ & 80 & $80(100)$ & - & - \\
\hline & & Experimental & 87 & $87(100)$ & 87 & $87(100)$ & - & - \\
\hline \multirow{4}{*}{ Coração } & \multirow{2}{*}{ Rapaz } & Controlo & 89 & & 89 & $82(92,1)$ & 0,94 & 0,332 \\
\hline & & Experimental & 71 & $66(93,0)$ & 72 & $72(100)$ & 3,37 & 0,028 \\
\hline & \multirow{2}{*}{ Rapariga } & Controlo & 79 & $76(96,2)$ & 80 & $80(100)$ & 1,39 & 0,120 \\
\hline & & Experimental & 87 & $86(98,9)$ & 87 & $87(100)$ & 0,00 & 1,000 \\
\hline \multirow{4}{*}{ Pele } & \multirow{2}{*}{ Rapaz } & Controlo & 90 & $77(85,6)$ & 90 & $75(83,3)$ & 0,04 & 0,837 \\
\hline & & Experimental & 71 & $47(66,2)$ & 72 & $68(94,4)$ & 16,37 & $<0,001$ \\
\hline & \multirow{2}{*}{ Rapariga } & Controlo & 79 & $66(83,5)$ & 80 & $79(98,8)$ & 9,63 & 0,001 \\
\hline & & Experimental & 87 & $74(85,1)$ & 87 & $86(98,9)$ & 9,40 & 0,001 \\
\hline \multirow{4}{*}{ Dentes } & \multirow{2}{*}{ Rapaz } & Controlo & 90 & $84(93,3)$ & 90 & $84(93,3)$ & 0,00 & 1,000 \\
\hline & & Experimental & 71 & $67(94,4)$ & 72 & $69(95,6)$ & 0,00 & 0,719 \\
\hline & \multirow{2}{*}{ Rapariga } & Controlo & 80 & $76(95,0)$ & 80 & $78(97,5)$ & 0,17 & 0,681 \\
\hline & & Experimental & 87 & $84(96,6)$ & 87 & $87(100)$ & 1,36 & 0,246 \\
\hline \multirow{4}{*}{ Sexualidade } & \multirow{2}{*}{ Rapaz } & Controlo & 89 & $65(73,0)$ & 90 & $68(75,6)$ & 0,05 & 0,735 \\
\hline & & Experimental & 71 & $50(70,4)$ & 72 & $67(93,1)$ & 10,84 & $<0,001$ \\
\hline & \multirow{2}{*}{ Rapariga } & Controlo & 79 & $42(53,2)$ & 80 & $58(72,5)$ & 5,57 & 0,014 \\
\hline & & Experimental & 84 & $45(53,6)$ & 87 & $85(97,7)$ & 43,27 & $<0,001$ \\
\hline \multirow{4}{*}{$\begin{array}{l}\text { Capacidade de } \\
\text { fazer desporto }\end{array}$} & \multirow[t]{2}{*}{ Rapaz } & Controlo & 90 & $83(92,2)$ & 90 & $82(91,1)$ & 0,00 & 1,000 \\
\hline & & Experime & 71 & $66(93,0)$ & 72 & $72(100)$ & 3,37 & 0,028 \\
\hline & \multirow{2}{*}{ Rapariga } & Controlo & 79 & $72(91,1)$ & 80 & $76(95,0)$ & 0,42 & 0,369 \\
\hline & & Experimental & 87 & $82(94,3)$ & 87 & $86(98,9)$ & 1,55 & 0,211 \\
\hline \multirow{4}{*}{$\begin{array}{l}\text { Saúde em } \\
\text { geral }\end{array}$} & Rapaz & Controlo & 91 & $85(93,4)$ & 91 & $85(93,4)$ & 0,00 & 1,000 \\
\hline & \multirow{3}{*}{ Rapariga } & Experi & 72 & $71(98,6)$ & 72 & $72(100)$ & 0,00 & 1,000 \\
\hline & & Controlo & 80 & $80(100)$ & 80 & $80(100)$ & - & - \\
\hline & & Experimental & 87 & $87(100)$ & 87 & $87(100)$ & - & . \\
\hline
\end{tabular}

Nota: $\%=$ percentagem de participantes a considerar o tabaco "prejudicial" ou "muito prejudicial (categorias agregadas) para o organismo. Neste caso, responder uma destas categorias de resposta era considerado o conhecimento adequado.

\section{Crenças sobre tabagismo}

Entre o pré e o pós-teste registaram-se, no grupo experimental, mudanças positivas em algumas crenças. A mudança positiva consistiu no aumento da discordância (quadro 2) ou concordância (quadro 3) com uma determinada crença, comparando a mudança positiva versus não mudança. Verificou-se um aumento da percentagem de alunos a discordar das seguintes afirmações: "Fumar é bom para emagrecer", tanto em rapazes (de $42,3 \%$ para $70,8 \% ; \mathrm{p}=0,001)$ como em raparigas $(54,0 \%$ para 77,0\%; $\mathrm{p}=0,002)$; "O tabaco ajuda a acalmar", tanto em rapazes (de 14,1\% para 58,3\%; $<<0,001$ ) como em raparigas (de 32,2\% para 65,1\%; p<0,001) e "Fumar alivia a tristeza", tanto em rapazes (de $25,4 \%$ para $45,8 \%$; $\mathrm{p}=0,014$ ) como em raparigas (de $42,5 \%$ para $66,7 \%$; $\mathrm{p}=0,002$ ). Analisando por sexo, verificou-se nas raparigas um aumento da discordância, entre o pré e o pós-teste, das crenças "A maioria dos adultos fuma" (de 16,1\% para 35,6\%; p=0,005); "Os fumadores têm mais amigos" (de $62,4 \%$ para $78,2 \%$ p=0,030) e "A maioria dos jovens fuma" (de 17,2\% para 33,7\%; $\mathrm{p}=0,015$ ). 


\section{PREVENÇÃO DO TABAGISMO NA ESCOLA}

Quadro 2.

Crenças sobre tabagismo, no pré e no pós-teste, por sexo, em que a mudança positiva corresponde à discordância

\begin{tabular}{|c|c|c|c|c|c|c|c|c|}
\hline \multirow[b]{2}{*}{ Variáveis } & \multirow[b]{2}{*}{ Sexo } & \multirow[b]{2}{*}{ Grupo } & \multicolumn{2}{|c|}{ Pré-teste } & \multicolumn{2}{|c|}{ Pós-teste } & \multicolumn{2}{|c|}{ Pré/Pós teste } \\
\hline & & & $\mathrm{N}$ & $\mathrm{n}(\%)$ & $\mathrm{N}$ & $\mathrm{n}(\%)$ & $x^{2}$ & $p$ \\
\hline \multirow{4}{*}{$\begin{array}{l}\text { Fumar é bom } \\
\text { Para } \\
\text { emagrecer }\end{array}$} & \multirow{2}{*}{ Rapaz } & Controlo & 90 & $35(38,9)$ & 90 & $42(46,7)$ & 0,82 & 0,366 \\
\hline & & Experimental & 71 & $30(42,3)$ & 72 & $51(70,8)$ & 10,75 & 0,001 \\
\hline & \multirow{2}{*}{ Rapariga } & Controlo & 80 & $41(51,3)$ & 80 & $38(47,5)$ & 0,10 & 0,752 \\
\hline & & Experimental & 87 & $47(54,0)$ & 87 & $67(77,0)$ & 9,18 & 0,002 \\
\hline \multirow{4}{*}{$\begin{array}{l}\text { O tabaco ajuda } \\
\text { a acalmar }\end{array}$} & \multirow{2}{*}{ Rapaz } & Controlo & 90 & $11(12,2)$ & 90 & $25(27,8)$ & 5,87 & 0,015 \\
\hline & & Experimental & 71 & $10(14,1)$ & 72 & $42(58,3)$ & 28,37 & $<0,001$ \\
\hline & \multirow{2}{*}{ Rapariga } & Controlo & 78 & $12(15,4)$ & 80 & $17(21,3)$ & 0,56 & 0,413 \\
\hline & & Experimental & 87 & $28(32,2)$ & 86 & $56(65,1)$ & 17,48 & $<0,001$ \\
\hline \multirow{4}{*}{$\begin{array}{l}\text { Não é } \\
\text { prejudicial } \\
\text { para a saúde } \\
\text { estar numa } \\
\text { sala com } \\
\text { fumadores }\end{array}$} & \multirow{2}{*}{ Rapaz } & Controlo & 90 & $60(66,7)$ & 89 & $68(76,4)$ & 1,63 & 0,185 \\
\hline & & Experimental & 72 & $52(72,2)$ & 72 & $57(79,2)$ & 0,60 & 0,437 \\
\hline & \multirow[b]{2}{*}{ Rapariga } & Controlo & 78 & $58(74,4)$ & 80 & $53(66,3)$ & 0,89 & 0,299 \\
\hline & & Experimental & 87 & $75(86,2)$ & 87 & $80(92,0)$ & 0,95 & 0,331 \\
\hline \multirow{4}{*}{$\begin{array}{l}\text { A maioria dos } \\
\text { adultos fuma }\end{array}$} & \multirow{2}{*}{ Rapaz } & Controlo & 90 & $8(8,9)$ & 90 & $25(27,8)$ & 9,50 & 0,002 \\
\hline & & Experimental & 72 & $18(25,0)$ & 72 & $25(34,7)$ & 1,19 & 0,275 \\
\hline & \multirow{2}{*}{ Rapariga } & Controlo & 80 & $14(17,5)$ & 80 & $10(12,5)$ & 0,44 & 0,507 \\
\hline & & Experimental & 87 & $14(16,1)$ & 87 & $31(35,6)$ & 7,67 & 0,005 \\
\hline \multirow{4}{*}{$\begin{array}{l}\text { Os fumadores } \\
\text { têm mais } \\
\text { amigos }\end{array}$} & \multirow{2}{*}{ Rapaz } & Controlo & 89 & $46(51,7)$ & 90 & $52(57,8)$ & 0,45 & 0,454 \\
\hline & & Experimental & 72 & $36(50,0)$ & 72 & $40(55,6)$ & 0,25 & 0,617 \\
\hline & \multirow{2}{*}{ Rapariga } & Controlo & 80 & $41(51,3)$ & 80 & $57(71,3)$ & 5,93 & 0,015 \\
\hline & & Experimental & 85 & $53(62,4)$ & 87 & $68(78,2)$ & 4,42 & 0,030 \\
\hline \multirow{4}{*}{$\begin{array}{l}\text { Os médicos } \\
\text { exageram } \\
\text { quando falam } \\
\text { dos malefícios } \\
\text { do tabaco }\end{array}$} & \multirow[t]{2}{*}{ Rapaz } & Controlo & 90 & $58(64,4)$ & 90 & $61(67,8)$ & 0,10 & 0,637 \\
\hline & & Experimental & 72 & $54(75,0)$ & 72 & $53(73,6)$ & 0,00 & 1,000 \\
\hline & \multirow[b]{2}{*}{ Rapariga } & Controlo & 80 & $61(76,3)$ & 80 & $59(73,8)$ & 0,03 & 0,855 \\
\hline & & Experimental & 87 & $72(82,8)$ & 87 & $71(81,6)$ & 0,00 & 1,000 \\
\hline & Rapaz & Controlo & 90 & $15(16,7)$ & 89 & $25(28,1)$ & 2,74 & 0,075 \\
\hline A maioria dos & & Experimental & 72 & $17(23,6)$ & 71 & $22(31,0)$ & 0,64 & 0,352 \\
\hline & Rapariga & Controlo & 79 & $6(7,6)$ & 80 & $7(8,8)$ & 0,00 & 1,000 \\
\hline & & Experimental & 87 & $15(17,2)$ & 86 & $29(33,7)$ & 5,36 & 0,015 \\
\hline Fumar só tem & Rapaz & Controlo & 90 & $37(41,1)$ & 90 & $34(37,8)$ & 0,09 & 0,760 \\
\hline consequências & & Experimental & 72 & $25(34,7)$ & 78 & $32(44,4)$ & 1,05 & 0,307 \\
\hline negativas na & Rapariga & Controlo & 80 & $34(42,5)$ & 79 & $29(36,7)$ & 0,34 & 0,518 \\
\hline $\begin{array}{l}\text { saúde se fumar } \\
\text { muitos anos }\end{array}$ & & Experimental & 87 & $41(47,1)$ & 87 & $46(52,9)$ & 0,37 & 0,544 \\
\hline Fumar é uma & Rapaz & Controlo & 89 & $50(56,2)$ & 89 & $55(61,8)$ & 0,37 & 0,542 \\
\hline boa maneira & & Experimental & 72 & $47(65,3)$ & 72 & $40(55,6)$ & 1,05 & 0,307 \\
\hline dos jovens & Rapariga & Controlo & 80 & $60(75,0)$ & 80 & $64(80,0)$ & 0,32 & 0,570 \\
\hline $\begin{array}{l}\text { mostrarem } \\
\text { independência }\end{array}$ & & Experimental & 87 & $68(78,2)$ & 87 & $64(73,6)$ & 0,28 & 0,595 \\
\hline & Rapaz & Controlo & 90 & $23(25,6)$ & 90 & $31(34,4)$ & 1,30 & 0,255 \\
\hline Fumar alivia a & & Experimental & 71 & $18(25,4)$ & 72 & $33(45,8)$ & 5,67 & 0,014 \\
\hline & Rapariga & Controlo & 80 & $24(30,0)$ & 80 & $33(41,3)$ & 1,74 & 0,186 \\
\hline & & Experimental & 87 & $37(42,5)$ & 87 & $58(66,7)$ & 9,27 & 0,002 \\
\hline
\end{tabular}




\section{Isabel Sousa}

\begin{tabular}{|c|c|c|c|c|c|c|c|c|}
\hline \multirow{4}{*}{$\begin{array}{l}\text { Fumar torna as } \\
\text { pessoas mais } \\
\text { bonitas/ } \\
\text { interessantes }\end{array}$} & Rapaz & Controlo & 89 & $67(75,3)$ & 90 & $67(74,4)$ & 0,00 & 1,000 \\
\hline & & Experimental & 72 & $60(83,3)$ & 72 & $58(80,6)$ & 0,05 & 0,829 \\
\hline & Rapariga & Controlo & 79 & $72(91,1)$ & 80 & $74(92,5)$ & 0,00 & 0,781 \\
\hline & & Experimental & 87 & $83(95,4)$ & 87 & $81(93,1)$ & 0,11 & 0,747 \\
\hline \multirow{4}{*}{$\begin{array}{l}\text { Fumar provoca } \\
\text { doenças sem } \\
\text { importância }\end{array}$} & Rapaz & Controlo & 90 & $71(78,9)$ & 90 & $75(83,3)$ & 0,33 & 0,568 \\
\hline & & Experimental & 72 & $57(79,2)$ & 72 & $59(81,9)$ & 0,04 & 0,834 \\
\hline & Rapariga & Controlo & 79 & $72(91,1)$ & 80 & $72(90,0)$ & 0,00 & 1,000 \\
\hline & & Experimental & 87 & $79(90,8)$ & 87 & $80(92,0)$ & 0,00 & 1,000 \\
\hline
\end{tabular}

Nota: \% = percentagem de alunos que discorda com a crença, sendo a discordância a mudança positiva.

Analisando a concordância como mudança positiva (quadro 3), verificou-se um aumento estatisticamente significativo entre os rapazes do grupo experimental, do pré para o pós-teste, nas crenças "Quem fuma tem uma pele envelhecida" (de 62,5\% para 83,4\%; p=0,008) e "As raparigas são mais sensíveis ao fumo do tabaco" (de 16,7\% para 34,7\%; $p=0,021$ ). 


\section{PREVENÇÃO DO TABAGISMO NA ESCOLA}

Quadro 3.

Crenças sobre tabagismo, no pré e no pós-teste, por sexo, em que a mudança positiva corresponde à concordância

\begin{tabular}{|c|c|c|c|c|c|c|c|c|}
\hline \multirow[b]{2}{*}{ Variáveis } & \multirow[b]{2}{*}{ Sexo } & \multirow[b]{2}{*}{ Grupo } & \multicolumn{2}{|c|}{ Pré-teste } & \multicolumn{2}{|c|}{ Pós-teste } & \multicolumn{2}{|c|}{ Pré/Pós teste } \\
\hline & & & $\mathrm{N}$ & n $(\%)$ & $\mathrm{N}$ & $\mathrm{n}(\%)$ & $x^{2}$ & $\mathrm{p}$ \\
\hline \multirow{4}{*}{$\begin{array}{l}\text { Quem fuma } \\
\text { tem } \\
\text { uma pele } \\
\text { envelhecida }\end{array}$} & \multirow{2}{*}{ Rapaz } & Controlo & 90 & $63(70,0)$ & 90 & $63(70,0)$ & 0,00 & 1,000 \\
\hline & & Experimental & 72 & $45(62,5)$ & 72 & $60(83,4)$ & 6,89 & 0,008 \\
\hline & \multirow{2}{*}{ Rapariga } & Controlo & 79 & $60(75,9)$ & 79 & $67(84,8)$ & 1,45 & 0,220 \\
\hline & & Experimental & 87 & $63(72,4)$ & 87 & $73(83,9)$ & 2,73 & 0,098 \\
\hline \multirow{4}{*}{$\begin{array}{l}\text { As raparigas } \\
\text { são mais } \\
\text { sensíveis ao } \\
\text { fumo do } \\
\text { tabaco }\end{array}$} & \multirow{2}{*}{ Rapaz } & Controlo & 90 & $15(16,7)$ & 90 & $10(11,1)$ & 0,74 & 0,389 \\
\hline & & Experimental & 72 & $12(16,7)$ & 72 & $25(34,7)$ & 5,24 & 0,021 \\
\hline & \multirow{2}{*}{ Rapariga } & Controlo & 80 & $16(20,0)$ & 80 & $14(17,5)$ & 0,04 & 0,840 \\
\hline & & Experimental & 87 & $24(27,6)$ & 87 & $29(33,3)$ & 0,43 & 0,510 \\
\hline \multirow{4}{*}{$\begin{array}{l}\text { O tabaco é } \\
\text { uma droga } \\
\text { muito viciante }\end{array}$} & \multirow{2}{*}{ Rapaz } & Controlo & 90 & $65(72,2)$ & 90 & $66(73,3)$ & 0,00 & 1,000 \\
\hline & & Experimental & 72 & $57(79,2)$ & 72 & $61(84,7)$ & 0,42 & 0,516 \\
\hline & \multirow{2}{*}{ Rapariga } & Controlo & 80 & $71(88,8)$ & 80 & $69(86,3)$ & 0,06 & 0,812 \\
\hline & & Experimental & 87 & $69(79,3)$ & 87 & $71(81,6)$ & 0,04 & 0,849 \\
\hline \multirow{4}{*}{$\begin{array}{l}\text { É prejudicial } \\
\text { para a saúde } \\
\text { estar ao lado } \\
\text { de alguém que } \\
\text { está a fumar }\end{array}$} & \multirow{2}{*}{ Rapaz } & Controlo & 90 & $40(44,4)$ & 90 & $51(56,7)$ & 2,22 & 0,136 \\
\hline & & Experimental & 71 & $43(60,6)$ & 70 & $49(70,0)$ & 1,00 & 0,290 \\
\hline & \multirow{2}{*}{ Rapariga } & Controlo & 80 & $52(65,0)$ & 79 & $53(67,1)$ & 0,01 & 0,867 \\
\hline & & Experimental & 87 & $58(66,7)$ & 87 & $67(77,0)$ & 1,82 & 0,177 \\
\hline \multirow{4}{*}{$\begin{array}{l}\text { Fumar durante } \\
\text { a gravidez } \\
\text { prejudica o } \\
\text { bebé }\end{array}$} & \multirow{2}{*}{ Rapaz } & Controlo & 88 & $74(84,1)$ & 89 & $78(87,6)$ & 0,21 & 0,525 \\
\hline & & Experimental & 72 & $66(91,7)$ & 72 & $63(87,5)$ & 0,30 & 0,587 \\
\hline & \multirow{2}{*}{ Rapariga } & Controlo & 80 & $78(97,5)$ & 80 & $76(95,0)$ & $0,17^{*}$ & 0,681 \\
\hline & & Experimental & 87 & $83(95,4)$ & 87 & $83(95,4)$ & $0,00^{*}$ & 1,000 \\
\hline \multirow{4}{*}{$\begin{array}{l}\text { Fumar é caro e } \\
\text { prejudica a } \\
\text { economia } \\
\text { familiar }\end{array}$} & \multirow[t]{2}{*}{ Rapaz } & Controlo & 89 & $67(75,3)$ & 89 & $75(84,3)$ & 1,71 & 0,191 \\
\hline & & Experimental & 71 & $63(88,7)$ & 72 & $61(84,7)$ & 0,21 & 0,623 \\
\hline & \multirow{2}{*}{ Rapariga } & Controlo & 79 & $68(86,1)$ & 80 & $72(90,0)$ & 0,27 & 0,474 \\
\hline & & Experimental & 86 & $74(86,0)$ & 87 & $77(88,5)$ & 0,07 & 0,655 \\
\hline \multirow{4}{*}{$\begin{array}{l}\text { Fumar } \\
\text { aumenta a } \\
\text { probabilidade } \\
\text { de ter cancro }\end{array}$} & \multirow[t]{2}{*}{ Rapaz } & Controlo & 90 & $74(82,2)$ & 90 & $75(83,3)$ & 0,00 & 1,000 \\
\hline & & Experimental & 72 & $64(88,9)$ & 72 & $63(87,5)$ & 0,00 & 1,000 \\
\hline & \multirow[t]{2}{*}{ Rapariga } & Controlo & 79 & $74(93,7)$ & 80 & $76(95,0)$ & & 0,746 \\
\hline & & Experimental & 87 & $80(92,0)$ & 87 & $83(95,4)$ & 0,39 & 0,535 \\
\hline
\end{tabular}

Nota: $\%$ = percentagem de alunos que concorda com a crença, sendo a concordância a mudança positiva.

*Teste de Fisher

\section{Experimentação de tabaco}

Entre os adolescentes que não tinham experimentado fumar tabaco no pré-teste, constatou-se que $23,1 \%$ dos rapazes e 20,8\% das raparigas do grupo de controlo declararam ter experimentado fumar no pós-teste, enquanto que no grupo experimental esta percentagem foi significativamente menor: $6,7 \%$ nos rapazes e 5,0\% nas raparigas; $p=0,026$ e $p=0,011$, respetivamente (quadro 4).

\section{Quadro 4.}


Experimentação de tabaco no pós-teste, por sexo

\begin{tabular}{|c|c|c|c|c|c|c|c|c|c|c|c|c|}
\hline \multirow{2}{*}{ Grupo } & \multicolumn{4}{|c|}{ Total } & \multicolumn{4}{|c|}{ Rapazes } & \multicolumn{4}{|c|}{ Raparigas } \\
\hline & $\mathrm{N}$ & $\mathrm{n}$ & $\%$ & $X^{2}(p)$ & $\mathrm{N}$ & $\mathrm{n}$ & $\%$ & $X^{2}(p)$ & $\mathrm{N}$ & $\mathrm{n}$ & $\%$ & $\mathrm{X}^{2}(\mathrm{p})$ \\
\hline Controlo & 105 & 23 & 21,9 & 11,56 & 52 & 12 & 23,1 & 4,97 & 53 & 11 & 20,8 & 6,44 \\
\hline Experimental & 105 & 6 & 5,7 & $(0,001)$ & 45 & 3 & 6,7 & $(0,026)$ & 60 & 3 & 5,0 & $(0,011)$ \\
\hline
\end{tabular}

Nota: $\%=$ percentagem de alunos que experimentaram fumar entre o pré e o pós-teste.

\section{Intenção de experimentar fumar tabaco}

Quanto à intenção de experimentar fumar tabaco no mês seguinte verificou-se que, no pós-teste, $23,3 \%$ das raparigas do grupo de controlo tencionavam experimentar no mês seguinte, enquanto que no grupo experimental apenas $6,8 \%$ tinham essa intenção. Nos rapazes, verificou-se uma redução da prevalência desta intenção no grupo experimental, entre o pré e o pós-teste, de 18,6\% para 15,9\% (sim ou talvez), acontecendo o contrário no grupo de controlo, onde se registou um aumento (de 8,4\% para $11,9 \%$ ), ainda que estas diferenças não sejam estatisticamente significativas (quadro 5).

\section{Quadro 5.}

Percentagem de jovens que não tendo experimentado fumar têm a intenção de experimentar fumar no mês seguinte, no pré e no pós-teste, por sexo

\begin{tabular}{|c|c|c|c|c|c|c|c|c|c|c|c|}
\hline & \multirow[b]{3}{*}{ Grupo } & \multicolumn{4}{|c|}{ Pré-teste } & \multicolumn{4}{|c|}{ Pós-teste } & \multirow{2}{*}{\multicolumn{2}{|c|}{$\begin{array}{c}\text { Pré/Pós } \\
\bar{T} \text { este }\end{array}$}} \\
\hline & & & Não & Talvez & $\mathrm{Sim}^{+}$ & & Não & Talvez & $\mathrm{Sim}^{+}$ & & \\
\hline & & $\mathrm{N}$ & $\mathrm{n}(\%)$ & $\mathrm{n}(\%)$ & $\mathrm{n}(\%)$ & $\mathrm{N}$ & $\mathrm{n}(\%)$ & $\mathrm{n}(\%)$ & $\mathrm{n}(\%)$ & $\mathrm{x}^{2}$ & $\mathrm{p}$ \\
\hline \multirow{2}{*}{ Rapaz } & Controlo & 48 & $44(91,7)$ & $3(6,3)$ & $1(2,1)$ & 42 & $37(88,1)$ & $5(11,9)$ & $0(0)$ & $1,66^{*}$ & 0,465 \\
\hline & Experimental & 43 & $35(81,4)$ & $8(18,6)$ & $0(0)$ & 44 & $37(84,1)$ & $7(15,9)$ & $0(0)$ & 0,00 & 0,961 \\
\hline \multirow{2}{*}{ Rapariga } & Controlo & 51 & $38(74,5)$ & $12(23,5)$ & $1(2,0)$ & 43 & $33(76,7)$ & $10(23,3)$ & $0(0)$ & $0,82 *$ & 1,000 \\
\hline & Experimental & 57 & $52(91,2)$ & $5(8,8)$ & $0(0)$ & 59 & $55(93,2)$ & $4(6,8)$ & $0(0)$ & 0,00 & 0,741 \\
\hline \multirow{2}{*}{ Total } & Controlo & 99 & $82(82,8)$ & $15(15,2)$ & $2(2,0)$ & 85 & $70(82,4)$ & $15(17,6)$ & $0(0)$ & 1,54 & 0,529 \\
\hline & Experimental & 100 & $87(87,0)$ & $13(13,0)$ & $0(0)$ & 103 & $92(89,3)$ & $11(10,7)$ & $0(0)$ & 0,09 & 0,768 \\
\hline
\end{tabular}

Nota: $\%$ = percentagem de alunos que têm intenção de experimentar fumar tabaco no mês seguinte, no pré e no pós-teste.

${ }^{+}$Não incluído no teste do Qui-Quadrado

*Teste de Fisher

No que se refere à intenção de experimentar fumar tabaco no ano seguinte (quadro 6), ainda que sem significância estatística, verificou-se uma subida no grupo de controlo, entre o pré e o pós-teste, de $22,4 \%$ para $28,2 \%$ (sim ou talvez), enquanto no grupo experimental se registou uma ligeira descida de $18,0 \%$ para $17,5 \%$. Esta intenção é menos acentuada nas raparigas, tendo-se verificado uma diminuição no grupo experimental, entre o pré e o pós-teste, de 14,0\% para 10,2\% (sim ou talvez), acontecendo o contrário no grupo de controlo, onde se registou um aumento da prevalência de alunas que tencionam vir a fumar de $31,4 \%$ para $37,2 \%$. 


\section{PREVENÇÃO DO TABAGISMO NA ESCOLA}

\section{Quadro 6.}

Percentagem de jovens que não tendo experimentado fumar têm a intenção de experimentar no ano seguinte, no pré e no pós-teste, por sexo

\begin{tabular}{|c|c|c|c|c|c|c|c|c|c|c|c|}
\hline & \multirow[b]{3}{*}{ Grupo } & \multirow[b]{3}{*}{$\mathrm{N}$} & \multicolumn{3}{|c|}{ Pré-teste } & \multicolumn{4}{|c|}{ Pós-teste } & \multirow{2}{*}{\multicolumn{2}{|c|}{$\begin{array}{c}\text { Pré/Pós } \\
\text { Teste }\end{array}$}} \\
\hline & & & Não & Talvez & $\mathrm{Sim}^{+}$ & & Não & Talvez & $\mathrm{Sim}^{+}$ & & \\
\hline & & & $\mathrm{n}$ & $\mathrm{n}(\%)$ & $\mathrm{n}(\%)$ & $\mathrm{N}$ & $\mathrm{n}(\%$ & $\mathrm{n}(\%)$ & $\mathrm{n}(\%)$ & $x^{2}$ & $\mathrm{p}$ \\
\hline \multirow{2}{*}{ Rapaz } & Controlo & 47 & 41 & $6(12,8)$ & $0(0)$ & 42 & 34 & $7(16,7)$ & $1(2,4)$ & $1,42^{*}$ & 0,5 \\
\hline & Experiment & 43 & 33 & 10 & $0(0)$ & 44 & 32 & 12 & $0(0)$ & 0,03 & 0,8 \\
\hline \multirow{2}{*}{$\begin{array}{l}\text { Rapari } \\
\text { ga }\end{array}$} & Controlo & 51 & 35 & 11 & 5 & 43 & 27 & 13 & $3(7,0)$ & $1,05^{*}$ & 0,6 \\
\hline & Experiment & 57 & 49 & $8(14,0)$ & $0(0)$ & 59 & 53 & $5(8,5)$ & $1(1,7)$ & $1,74^{*}$ & 0,3 \\
\hline \multirow{2}{*}{ Total } & Controlo & 98 & 76 & 17 & 5 & 85 & 61 & 20 & $4(4,7)$ & $1,13^{*}$ & 0,5 \\
\hline & Experiment & 10 & 82 & 18 & $0(0)$ & 10 & 85 & 17 & $1(1,0)$ & $0,99 *$ & 0,9 \\
\hline
\end{tabular}

Nota: \% = percentagem de alunos que têm intenção de experimentar fumar tabaco no ano seguinte, no pré e no pós-teste.

${ }^{+}$Não incluído no teste do Qui-Quadrado

*Teste de Fisher

Entre os adolescentes que nunca experimentaram fumar, ainda que os resultados não atinjam significância estatística, verificou-se no grupo experimental, entre o pré e o pós-teste, uma ligeira subida da prevalência de rapazes a reportar a intenção de experimentar fumar antes dos 18 anos, de $32,5 \%$ para $34,0 \%$ (sim ou talvez), enquanto nas raparigas se registou uma descida de $24,6 \%$ para $22,0 \%$ (quadro 7). O mesmo se verificou nos rapazes do grupo de controlo, havendo um aumento da intenção de experimentação de $26,5 \%$ para $31,0 \%$ (sim ou talvez), enquanto nas raparigas se registou uma descida de $54,0 \%$ para $39,6 \%$.

\section{Quadro 7.}

Percentagem de jovens que não tendo experimentado fumar têm a intenção de experimentar antes dos 18 anos, no pré e no pós-teste, por sexo

\begin{tabular}{|c|c|c|c|c|c|c|c|c|c|c|c|}
\hline & \multirow[b]{3}{*}{ Grupo } & \multicolumn{4}{|c|}{ Pré-teste } & \multicolumn{4}{|c|}{ Pós-teste } & \multirow{2}{*}{\multicolumn{2}{|c|}{$\begin{array}{c}\text { Pré/Pós } \\
\text { Teste }\end{array}$}} \\
\hline & & & Não & Talvez & Sim & & Não & Talvez & Sim & & \\
\hline & & $\mathrm{N}$ & $\mathrm{n}$ & $\mathrm{n}(\%)$ & $\mathrm{n}(\%)$ & $\mathrm{N}$ & $\mathrm{n}$ & $\mathrm{n}$ & $\mathrm{n}(\%)$ & $\mathrm{x}^{2}$ & $\mathrm{p}$ \\
\hline \multirow{2}{*}{ Rapaz } & Controlo & 49 & 36 & $5(10,2)$ & $8(16,3)$ & 42 & 29 & $7(16,7)$ & $6(14,3)$ & 0,84 & 0,657 \\
\hline & Experime & 43 & 29 & $9(20,9)$ & $5(11,6)$ & 44 & 29 & 13 & $2(4,5)$ & 1,96 & 0,403 \\
\hline \multirow{2}{*}{ Rapariga } & Controlo & 50 & 23 & 15 & 12 & 43 & 26 & $7(16,3)$ & 10 & 2,76 & 0,251 \\
\hline & Experime & 57 & 43 & 13 & $1(1,8)$ & 59 & 46 & 11 & $2(3,4)$ & 0,66 & 0,753 \\
\hline \multirow{2}{*}{ Total } & Controlo & 99 & 59 & 20 & 20 & 85 & 55 & 14 & 16 & 0,58 & 0,748 \\
\hline & Experime & 10 & 72 & 22 & $6(6,0)$ & 103 & 75 & 24 & $4(3,9)$ & 0,50 & 0,777 \\
\hline
\end{tabular}

Nota: $\%$ = percentagem de alunos que têm intenção de experimentar fumar tabaco antes dos 18 anos, no pré e no pós-teste.

*Teste de Fisher

Ainda que sem significância estatística, a prevalência de alunos que têm a intenção de experimentar fumar depois dos 18 anos, entre o pré e o pós-teste, diminuiu nas raparigas em ambos os grupos de $34,5 \%$ para $33,4 \%$ (sim ou talvez), no grupo experimental e de $62,7 \%$ para $58,1 \%$ no grupo de controlo e aumentou nos rapazes do grupo de controlo (de 42,0\% para 45,5\%) (quadro 8). 


\section{Isabel Sousa}

\section{Quadro 8.}

Percentagem de jovens que não tendo experimentado fumar têm a intenção de experimentar depois dos 18 anos, no pré e no pós-teste, por sexo

\begin{tabular}{|c|c|c|c|c|c|c|c|c|c|c|c|}
\hline & \multirow[b]{3}{*}{ Grupo } & \multirow[b]{3}{*}{$\mathrm{N}$} & \multicolumn{3}{|c|}{ Pré-teste } & \multicolumn{4}{|c|}{ Pós-teste } & \multirow{2}{*}{\multicolumn{2}{|c|}{$\begin{array}{l}\text { Pré/Pós } \\
\text { Teste }\end{array}$}} \\
\hline & & & Não & Talvez & Sim & & Não & Talvez & Sim & & \\
\hline & & & $\mathrm{n}(\%)$ & $\mathrm{n}(\%)$ & $\mathrm{n}(\%)$ & $\mathrm{N}$ & $\mathrm{n}(\%)$ & $\mathrm{n}(\%)$ & $\mathrm{n}(\%)$ & $x^{2}$ & $\mathrm{p}$ \\
\hline \multirow{2}{*}{ Rapaz } & Controlo & 50 & 29 & 13 & $8(16,0)$ & 44 & 24 & 11 & $9(20,5)$ & 0,32 & 0,854 \\
\hline & Experimental & 44 & 24 & 11 & $9(20,5)$ & 46 & 25 & 14 & $7(15,2)$ & 0,59 & 0,746 \\
\hline \multirow{2}{*}{ Rapariga } & Controlo & 51 & 19 & 15 & 17 & 43 & 18 & $9(20,9)$ & $16(37,2)$ & 0,88 & 0,643 \\
\hline & Experimental & 58 & 38 & 17 & $3(5,2)$ & 60 & 40 & 16 & $4(6,7)$ & $0,26^{*}$ & 0,954 \\
\hline \multirow{2}{*}{ Total } & Controlo & 101 & 48 & 28 & 25 & 87 & 42 & 20 & $25(28,7)$ & 0,70 & 0,707 \\
\hline & Experimental & 102 & 62 & 28 & 12 & 106 & 65 & 30 & $11(10,4)$ & 0,11 & 0,948 \\
\hline
\end{tabular}

Nota: $\%$ = percentagem de alunos que têm intenção de experimentar fumar tabaco depois dos 18 anos, no pré e no pós-teste.

*Teste de Fisher

\section{Iniciação do consumo de tabaco}

Analisando a iniciação do consumo de tabaco (quadro 9), verificou-se que 9,4\% dos alunos do grupo de controlo iniciaram o consumo de tabaco entre o pré e o pós-teste, enquanto no grupo experimental apenas $2,1 \%$ o fizeram $(\mathrm{p}=0,011)$. Analisando por sexo, verificou-se que $9,0 \%$ dos rapazes do grupo de controlo iniciaram o consumo de tabaco, enquanto que no grupo experimental iniciaram apenas 3,3\%. Nas raparigas, 9,9\% do grupo de controlo reportaram ter iniciado o consumo de tabaco no pós-teste, enquanto no grupo experimental apenas $1,2 \%$ o fizeram $(\mathrm{p}=0,007)$.

\section{Quadro 9.}

Iniciação do consumo de tabaco no pós-teste, por sexo

\begin{tabular}{|c|c|c|c|c|c|c|c|c|c|c|c|c|}
\hline \multirow{2}{*}{ Grupo } & \multicolumn{4}{|c|}{ Total } & \multicolumn{4}{|c|}{ Rapazes } & \multicolumn{4}{|c|}{ Raparigas } \\
\hline & $\mathrm{N}$ & $\mathrm{n}$ & $\%$ & $X^{2}(p)$ & $\mathrm{N}$ & $\mathrm{n}$ & $\%$ & $X^{2}(p)$ & $\mathrm{N}$ & $\mathrm{n}$ & $\%$ & $X^{2}(p)$ \\
\hline Controlo & 105 & 14 & 9,4 & 7,165 & 52 & 7 & 9,0 & 1,834 & 53 & 7 & 9,9 & $5,819 *$ \\
\hline Experimental & 105 & 3 & 2,1 & $(0,011)$ & 45 & 2 & 3,3 & $(0,298)^{*}$ & 60 & 1 & 1,2 & $(0,007)$ \\
\hline
\end{tabular}

Nota: $\%=$ percentagem de alunos que iniciaram o consumo de tabaco entre o pré e o pós-teste.

*Teste de Fisher

\section{Consumo de tabaco}

Verificou-se uma diminuição do consumo de tabaco no grupo experimental, ainda que sem significância estatística, entre o pré e o pós-teste de $6,9 \%$ para $5,7 \%$ (ocasional e regular), enquanto no grupo de controlo se registou um aumento de 11,1\% para 17,6\% (quadro 10). No que diz respeito aos rapazes, verificou-se uma diminuição do consumo no grupo experimental, sem significância estatística, entre o pré e o pós-teste de 9,9\% para 5,6\% (ocasional e regular), enquanto no grupo de controlo ocorreu um aumento de $13,2 \%$ para $18,7 \%$. Nas raparigas, constatou-se um aumento ligeiro do consumo no grupo experimental, de $4,5 \%$ para $5,7 \%$ (ocasional e regular), enquanto no grupo de controlo ocorreu um aumento de $8,8 \%$ para $16,3 \%$. 


\section{PREVENÇÃO DO TABAGISMO NA ESCOLA}

\section{Quadro 10.}

Consumo de tabaco, no pré e no pós-teste, por sexo

\begin{tabular}{|c|c|c|c|c|c|c|c|c|c|c|c|}
\hline \multirow[b]{3}{*}{ Sexo } & \multirow[b]{3}{*}{ Grupo } & \multirow[b]{3}{*}{$\mathrm{N}$} & \multicolumn{3}{|c|}{ Pré-teste } & \multirow[b]{3}{*}{$\mathrm{N}$} & \multicolumn{3}{|c|}{ Pós-teste } & \multirow{2}{*}{\multicolumn{2}{|c|}{$\begin{array}{c}\text { Pré/Pós } \\
\text { Teste }\end{array}$}} \\
\hline & & & Não & Ocasional & Regular $^{+}$ & & Não & Ocasional & Regular $^{+}$ & & \\
\hline & & & $\mathrm{n}(\%)$ & $\mathrm{n}(\%)$ & $\mathrm{n}(\%)$ & & $\mathrm{n}(\%)$ & $\mathrm{n}(\%)$ & $\mathrm{n}(\%)$ & $x^{2}$ & $\mathrm{p}$ \\
\hline Rapaz & Experimental & 71 & $64(90,1)$ & $0(0,0)$ & $7(9,9)$ & 72 & $68(94,4)$ & $1(1,4)$ & $3(4,2)$ & $2,58 *$ & 0,266 \\
\hline Rapariga & Controlo & 80 & $73(91,3)$ & $2(2,5)$ & $5(6,3)$ & 80 & $67(83,8)$ & $6(7,5)$ & $7(8,8)$ & $2,50 *$ & 0,276 \\
\hline \multirow{2}{*}{ Total } & Controlo & 171 & $152(88,9)$ & $2(1,2)$ & $17(9,9)$ & 171 & $141(82,5)$ & $9(5,3)$ & $21(12,3)$ & 5,22 & 0,072 \\
\hline & Experimental & 158 & $147(93,0)$ & $1(0,6)$ & $10(6,3)$ & 159 & $150(94,3)$ & $2(1,3)$ & $7(4,4)$ & 0,97 & 0,656 \\
\hline
\end{tabular}

Nota: $\%=$ percentagem de alunos que fumam tabaco no pré e no pós-teste.

${ }^{+}$Agregação (Ocasional e regular) no Teste do Qui-Quadrado

* Teste de Fisher

\section{DISCUSSÃO}

O programa "SmokeOut-II" revelou eficácia na melhoria dos conhecimentos e na mudança positiva de algumas crenças sobre o tabagismo. Esta associação foi já encontrada em outros programas de prevenção que mostraram uma melhoria do comportamento relativo ao tabagismo na sequência do investimento nos conhecimentos dos alunos (Nădăşan et al., 2015; Kanicka et al., 2013a). Além disso, verificou-se uma redução na experimentação e na intenção de vir a experimentar fumar tabaco, em ambos os sexos, bem como uma diminuição da prevalência do consumo regular de tabaco, a curto prazo. O programa parece ter contribuído para que os alunos do grupo experimental adquirissem maior consciência de que fumar tem consequências graves no organismo e na saúde, a curto e médio prazo, bem como na aparência dos fumadores. Os alunos sujeitos à intervenção do programa percecionaram que o consumo de tabaco não é um meio saudável e eficaz para emagrecer ou para acalmar estados de ansiedade, e que as consequências de fumar e/ou estar exposto ao fumo ambiental do tabaco são extremamente nefastas à saúde e prejudicam o ambiente. Estudos com adolescentes mostraram que intervenções que tiveram como propósito aumentar o nível de informação sobre as consequências negativas de um comportamento tiveram um efeito positivo no evitamento futuro desse comportamento por parte dos jovens (Müller-Riemenschneider et al., 2008; Vitória et al., 2011; Kanicka, Poniatowski, \& Szpak, 2013b).

O programa parece também ter tido um efeito protetor relativamente à iniciação do consumo de tabaco nas raparigas, salientando-se a diminuição do consumo regular nos rapazes, para além de promover perspicuidade na perceção de que a maioria dos jovens não fuma e que fumar não está na moda, bem como a tomada de consciência dos custos associados à compra de tabaco. Assim, o Programa "SmokeOut-II" constitui uma ferramenta importante para trabalhar a temática do tabagismo em contexto escolar, disponibilizando materiais variados e adaptados ao currículo, sendo um recurso facilitador, transversal e multidisciplinar que envolve a escola, a família, a comunidade e a sociedade, evidenciando eficácia na prevenção do tabagismo.

Contudo, este estudo apresenta limitações que devem ser discutidas: o reduzido tamanho da amostra e o facto de os dados serem provenientes apenas de uma região (um distrito do Norte de Portugal) implica prudência quanto à generalização de dados. Uma amostra maior permitiria análises estatísticas com maior poder estatístico. Seria importante desenvolver mais estudos implementando este programa em escolas inseridas em diferentes bairros socioeconómicos e diferentes regiões do país. 


\section{Isabel Sousa}

É necessário investigar se estes resultados persistem no tempo para avaliar a eficácia do programa a longo prazo. Se for demonstrada a sua efetividade, a sua implementação a nível nacional nas escolas do Ensino Básico deverá ser incentivada, de forma a promover uma intervenção preventiva global do consumo de tabaco em adolescentes.

\section{AGRADECIMENTOS}

A autora agradece o tratamento estatístico dos dados pelo Professor Doutor José Cunha Machado e o contributo na revisão científica do manuscrito ao Professor Doutor José Precioso e à Doutora Catarina Samorinha, bem como a disponibilidade de todos os participantes no estudo.

\section{FINANCIAMENTO}

Este trabalho foi financiado por Fundos Nacionais através da FCT (Fundação para a Ciência e a Tecnologia), pela Bolsa de Doutoramento SFRH/BD/125425/2016 e cofinanciado pelo Fundo Europeu de Desenvolvimento Regional (FEDER) através do COMPETE 2020 - Programa Operacional Competitividade e Internacionalização (POCI) no âmbito do CIEC (Centro de Investigação em Estudos da Criança da Universidade do Minho) com a referência POCI-01-0145-FEDER-007562.

\section{REFERÊNCIAS}

Andersen, A., Krølner, R., Bast, L.S., Thygesen, L. C., \& Due, P. (2015). Effects of the X:IT smoking intervention: a school-based cluster randomized trial. International Journal of Epidemiology, 44, 1900-1908. doi:10.1093/ije/dyv145

Currie, C., Gabhainn, S., Godeau, E., Roberts, C., Smith, R., Currie, D., ... Barnekow, V. (2008). Inequalities in young people's health. Health Behaviour in School-aged Children (HBSC): international report from the 2005/2006 survey. Edinburgh: HBSC International Coordinating Centre.

Currie, C., Hurrelmann, K., Settertobulte, W., Smith, R., \& Todd, J. (2000). Health and Health Behaviour among Young People. Study: International report from the 1997/1998 survey. Copenhagen: World Health Organization Regional Office for Europe.

Currie, C., Hurrelmann, K., Settertobulte, W., Smith, R., \& Todd, J. (2004). Young People's health in context. Health Behaviour in School-aged Children (HBSC). Study: International report from the 2001/2002 survey. Copenhagen: World Health Organization Regional Office for Europe.

Currie, C., Zanotti, C., Morgan, A., Currie, M.L., Roberts, C., Samdal, O., Smith, O.R.F., \& Barnekow, V. (2012). Social determinants of health and well-being among young people. Health Behaviour in School-aged Children (HBSC). Study: International report from the 2009/2010 survey. Copenhagen: World Health Organization Regional Office for Europe.

Fishbein, M., \& Ajzen, I. (1975). Belief, Attitude, Intention, and Behavior: An Introduction to Theory and Research. Reading, MA: Addison-Wesley.

Inchley, J., Currie, D., Young, T., Samdal, O., Torsheim, T., Augustson, L., Mathison, F., AlemanDiaz, A., Molcho, M., Webwe, M., \& Barnekow, V. (2016). Growing up unequal: gender and socioeconomic differences in young people's health and well-being. Health Behaviour in School- 


\section{PREVENÇÃO DO TABAGISMO NA ESCOLA}

aged Children (HBSC) study: international report from the 2013/2014 survey. Copenhagen, World Health Organization Regional Office for Europe.

Kanicka, M., Poniatowski, B., Szpak, A., \& Owoc, A. (2013a). Differences in the effects of antitobacco health education programme in the areas of knowledge, attitude and behaviour, with respect to nicotinism among boys and girls. Annals of Agricultural and Environmental Medicine, 20(1), 173-177.

Kanicka, M., Poniatowski, B., Szpak, A., \& Owoc, A. (2013b). Effect of an anti-tobacco programme of health education on changes in health behaviours among junior high school adolescents in Białystok, Poland. Annals of Agricultural and Environmental Medicine, 20(1), 167-172.

Mercken, L. Moore, L., Crone, M.R., De Vries, H., De Bourdeaudhuij, I., Lien, N., Fagiano, F., Vitória, P.D., \& Van Lenthe, F.J. (2012). The effectiveness of school-based smoking prevention interventions among low and high SES European teenagers. Health Education Research, 27(3), 459-469.

Müller-Riemenschneider, F., Bockelbrink, A., Reinhold, T., Rasch, A., Greiner, W., \& Willich, S.N. (2008). Long-term effectiveness of behavioural interventions to prevent smoking among children and youth. Tobacco Control, 17, 301-312. doi:10.1136/tc.2007.024281

Nădăşan, V., Chirvăsuţă, R., Ábrám, Z., \& Mihăicuţă, Ş. (2015). Types of Interventions for Smoking Prevention and Cessation in Children and Adolescents. Pneumologia, 64(3), 58-62.

Precioso, J. (1999). Não fumar é o que está a dar. Lisboa: Instituto de Inovação Educacional.

Precioso, J. (2001). Educação para a prevenção do comportamento de fumar. Avaliação de uma intervenção pedagógica no $3^{\circ}$ Ciclo do Ensino Básico (tese de doutoramento). Universidade do Minho, Braga.

Precioso, J., \& Samorinha, C. (2014). Fatores associados com o consumo de tabaco em jovens escolarizados portugueses, por sexo: Pensar e agir, global, local e sistematicamente contra o tabaco (Relatório de investigação). Braga: Associação para a Prevenção e Tratamento do Tabagismo de Braga. Centro de Investigação em Estudos da Criança - Universidade do Minho.

Precioso, J., Reis, MF., Sousa, I., Samorinha, C., Sousa, C., Correia, C., Antunes, H., Macedo, M., \& Machado, J. (2014). SmokeOut II (3º ciclo). Programa de Prevenção do Consumo de Tabaco. Braga: Associação para a Prevenção e Tratamento do Tabagismo de Braga (APTTB) \& Centro de Investigação em Estudos da Criança - Universidade do Minho (CIEC).

Precioso, J., Samorinha, C., \& Macedo, M. (2016). A prevenção do tabagismo em meio escolar: teoria e prática. In: J. A. Garcia del Castillo \& P. C. Dias (Eds.), Estudos sobre o Tabaco: Contributos para a Prática (pp.83-107). Braga: Axioma - Publicações da Faculdade de Filosofia.

Thomas, R.E., McLellan, J., \& Perera, R. (2013). School-based programmes for preventing smoking. Cochrane Database of Systematic Reviews, Issue 4. Art. No.: CD001293. doi: 10.1002/14651858.CD001293.pub3

U.S. Department of Health and Human Services [USDHHS], Centers for Disease Control and Prevention, National Center for Chronic Disease Prevention and Health Promotion, Office on Smoking and Health. (2012). Preventing tobacco use among youth and young adults: A report of the Surgeon General. Acedido a 28 de janeiro, 2018, em http://www.surgeongeneral.gov/library/reports/preventing-youth-tobacco-use/full-report

U.S. Department of Health and Human Services [USDHHS]. Centers for Disease Control and Prevention, National Center for Chronic Disease Prevention and Health Promotion, Office on Smoking and Health. (2014). The Health Consequences of Smoking: 50 Years of Progress. A Report of the Surgeon General. Atlanta, GA: U.S. Department of Health and Human Services, Centers for Disease Control and Prevention, National Center for Chronic Disease Prevention and Health Promotion, Office on Smoking and Health. 


\section{Isabel Sousa}

Vitória, P., Raposo, C., Peixoto, F. Carvalho, A., \& Clemente, M. (2001). "Querer é poder II” Programa de Prevenção do Tabagismo para o $3^{\circ}$ Ciclo do Ensino Básico - Manual do professor. Lisboa: Conselho de Prevenção do Tabagismo, Ministério da Saúde.

Vitória, P., Raposo, C., Peixoto, F., \& Clemente, M. (2000). "Querer é Poder I" - Programa de Prevenção do Tabagismo para o $3^{\circ}$ Ciclo do Ensino Básico - Manual do Professor. Lisboa: Conselho de Prevenção do Tabagismo, Ministério da Saúde.

Vitória, P., Silva, S., \& De Vries, H. (2011). Avaliação longitudinal de um programa de prevenção do tabagismo para adolescentes. Rev Saúde Pública, 45(2), 343-354.

World Health Organization. (2017). WHO report on the global tobacco epidemic, 2017: monitoring tobacco use and prevention policies. Geneva: World Health Organization. 